\title{
Research Methods for Studying Narrative Identity: A Primer
}

\section{This manuscript is currently in press at Social Psychological and Personality Science (as of January, 2017)}

\section{AUTHOR INFORMATION}

\author{
Jonathan M. Adler \\ Olin College of Engineering \\ jadler@olin.edu
}

Jonathan M. Adler is an associate professor of psychology at Olin College of Engineering. He is also an Associate Editor of the Journal of Personality, on the editorial board of Journal of Research in Personality, and Chief Scientific Officer of Health Story Collaborative. His research examines the dynamic relationships between adult identity development and psychological wellbeing.

William L. Dunlop

University of California at Riverside

william.dunlop@ucr.edu

William L. Dunlop is an assistant professor of personality psychology at the University of California, Riverside. His research examines personality, self, and identity using both idiographic and nomothetic approaches.

\author{
Robyn Fivush \\ Emory University \\ psyrf@emory.edu
}

Robyn Fivush is the Samuel Candler Dobbs Professor of Psychology at Emory University, Associated Faculty with the Department of Women's Studies, and a Senior Fellow in the Center for the Study of Law and Religion. Her research focuses on the social construction of autobiographical memory and the relations among memory, narrative, identity, trauma, and coping.

\author{
Jennifer P. Lilgendahl \\ Haverford College \\ jlilgend@haverford.edu
}

Jennifer Pals Lilgendahl is an associate professor of psychology at Haverford College. Her research examines processes of self-definition and identity construction in adolescence and adulthood, with a specific focus on how people connect memories of past events to the present self through the narration of a life story.

Jennifer Lodi-Smith

Canisius College 
lodismij@canisius.edu

Jennifer Lodi-Smith is an assistant professor of psychology at Canisius College. Her program of research uses mixed methods to understand developmental processes in the content and clarity of individual identity. She is a Member-At-Large for the Association for Research in Personality and serves on the editorial board for the Journal of Personality and Social Psychology: Personality Processes and Individual Differences.

\author{
Dan P. McAdams \\ Northwestern University \\ dmca@northwestern.edu
}

Dan P. McAdams is the Henry Wade Rogers Professor of Psychology at Northwestern University, Professor of Human Development and Social Policy, and the Director of the Foley Center for the Study of Lives. A personality and life-span developmental psychologist, McAdams is the author of The Art and Science of Personality Development (Guilford Press, 2015).

\author{
Kate C. McLean \\ Western Washington University \\ Kate.McLean@wwu.edu
}

Kate C. McLean is a professor of psychology at Western Washington University. Her research focuses on adolescent and emerging adult identity development. She is an Associate Editor for the Journal of Personality and Social Psychology: Personality Processes and Individual Differences and the Journal of Research in Personality.

\author{
Monisha Pasupathi \\ University of Utah \\ monisha.pasupathi@psych.utah.edu
}

Monisha Pasupathi is professor of developmental psychology at the University of Utah. Her $\mathrm{NIH}$-funded research program examines narrative as a process for self and social development across the lifespan. She has served as an Associate Editor for the Journal of Personality, a guest editor for Memory, and on the editorial boards of Developmental Psychology, Psychology and Aging, and the Journal of Emerging Adulthood.

\author{
Moin Syed \\ University of Minnesota, Twin Cities \\ moin@umn.edu
}

Moin Syed is an associate professor of psychology at the University of Minnesota, Twin Cities. His research is broadly concerned with identity and personality development among ethnically and culturally-diverse adolescents and emerging adults. He is co-Editor of the Oxford Handbook of Identity Development, and Editor of Emerging Adulthood. 


\begin{abstract}
This paper provides a primer for researchers seeking an introduction to quantitative narrative research methods. It represents a consensus document of most common practices used by the coauthors. Key elements of conducting narrative research (e.g., asking narrative questions, designing narrative prompts, collecting narratives, coding narratives) are discussed, along with limitations to this approach and future directions.
\end{abstract}


Research in wide-ranging sub-fields of psychology have capitalized on the power of narratives as a methodological tool, examining diverse topics such as psychotherapy (Adler, 2012), alcoholism (Dunlop \& Tracy, 2013), gender (Grysman, Merrill, \& Fivush, 2016), mid-life development (Pals, 2006), personality change (Lodi-Smith, et al., 2009), generativity (McAdams, 2006a), family processes (McLean, 2015), emotion regulation (Pasupathi, et al., 2015), ethnic identity development (Syed \& Azmita, 2010), meaning-making (McLean \& Thorne, 2003), adult attachment (Waters, Brockmeyer, \& Crowell, 2013), physical and mental health (Adler et al., 2015), political orientation (McAdams et al., 2008), and self-regulation (McAdams, Hanek, \& Dadabo, 2013). Personality psychologists have made especially strong contributions to this methodological approach in work on narrative identity, the story of the self that weaves together the reconstructed past, the perceived present, and the imagined future, providing the individual with a sense of unity and meaning (e.g., McAdams, 1995; McAdams \& McLean, 2013; McAdams \& Pals, 2006; Singer, 2004).

Despite this widespread use of narrative, social and personality psychologists still have many questions about what narrative is and how to use it as a tool. Indeed, it seems a critical barrier to researchers' use of narrative methods is not lack of interest, but lack of information. Thus, we draw on our collective knowledge as narrative researchers to describe common approaches to quantitative narrative research as a primer for scholars new to the field. We focus on four key aspects of narrative methods: asking narrative questions, designing narrative prompts, collecting narratives, and coding narratives (see Figure 1 for an overview). Although we primarily employ examples of narrative methods in the study of narrative identity, we also interweave examples from other domains to highlight the broad applicability of narrative. 
Importantly, this primer should be seen as a starting point for those interested in beginning narrative research, and neither an exhaustive tutorial nor a detailing of advanced topics.

\section{Theoretical Frame}

The theoretical rationale for using narrative methods has been elucidated elsewhere (e.g., Adler et al., 2016; Habermas \& Reese, 2015; McAdams \& McLean, 2013; McLean \& Syed, 2015); here, we address two common critiques of this approach: that the stories people tell about their lives are not necessarily true or accurate, and that narrative methods are merely laborintensive self-report instruments. Both criticisms miss the essential point about narratives.

First, like all autobiographical memories, narratives of personal experiences are dynamically reconstructed representations of events. Each time a memory is recalled, the retrieval process is a complex interaction between the internal neural context and external sociocultural context, modulated by the functions that remembering serves in that moment. Thus, narratives are deeply idiographic, dynamic reflections of how individuals recall their experiences, and serve context-specific functions. The purpose of narrative research is to understand these functions and their relationships with other correlates and outcomes, not the veracity of the memories. Narratives are subjective constructions that have objective impacts. For example, individual differences in autobiographical narratives are associated with the successful maintenance of sobriety among alcoholics (Dunlop \& Tracy, 2013) and the desistence from crime among ex-convicts (Maruna, 2001), and individual differences in the trajectory of narrative development precede changes in mental health among psychotherapy clients (Adler, 2012).

Second, narratives are not simply converging measures of identity; narratives are constitutive of identity, in that how we make sense of our experiences and who we perceive 
ourselves to be are reciprocally related across development (McAdams \& Pals, 2006; McLean, Pasupathi, \& Pals, 2007). Moreover, because narratives are deeply embedded in sociocultural interactions across the life course, how individuals story their lives reflects both explicit efforts at meaning-making, as well as implicit modes of being in the world (Fivush \& Merrill, 2016). Thus, narrative approaches to identity are not reducible to self-report measures, but rather represent a different level of analysis that assesses subjectivity in unique ways (Adler et al., 2016).

Finally, narratives allow researchers to ethically and meaningfully understand lived experiences in context (Fivush, 2010). Appreciating the voices and experiences of participants can be done by examining the dynamic and contextualized nature of stories, often accomplished via qualitative inquiry (e.g., Josselson, 2009; Hammack, 2008), as well as in nomothetic, hypothesis testing, or exploratory quantitative approaches. We focus on the quantitative approach in this primer because we most frequently receive questions about that approach.

\section{Asking Narrative Questions}

Studies that use narrative data ought to be designed to ask questions that narratives are especially well-suited to address. At its core, research using narrative methods is concerned with meaning-making. The empirical study of narrative is therefore a science of subjectivity, one that employs the tools of science for examining issues of personal meaning. Narrative questions are concerned with the explicit content of stories (e.g., emotional language), the implicit content of stories (e.g., themes), and/or the structural aspects of stories (e.g., coherence). Narrative methods are particularly well-situated to examine meaning-making processes that concern the self. Other approaches for examining meaning and identity may tap the extent to which participants feel their lives are meaningful, but in crafting personal narratives, participants demonstrate it. 
Narrative methods therefore allow for the enactment of meaning, rather than a report of the perception of meaningfulness.

The diversity and breadth of the field is certainly beneficial, but it has also led to some diffusion and redundancy. Recently, narrative scholars have begun to work towards providing some structure to the range of narrative variables that have been examined in relation to personality. McAdams and McLean (2013) identified six commonly-examined narrative constructs, highlighting those variables that have received substantial attention. Adler and colleagues (2016) developed an organizational framework for narrative variables related to psychological well-being and classified them into four categories: motivational themes, affective themes, themes of integrative meaning, and structural aspects of narratives. Table 1 provides an overview of these overlapping systems.

In the service of building a cumulative science of narrative identity, we recommend that researchers explicitly situate their investigations in the context of these organizational systems and the narrative variables listed in Table 1, when appropriate. That said, these proposed organizational structures are not a final taxonomy, and there is still much landscape to be filled in. For example, the focus on associations with well-being may have narrowed the landscape of variables examined. While there is no single compendium of existing coding systems, many of the most commonly used systems are presented in the reviews by McAdams and McLean (2013) and Adler and colleagues (2016), and are also summarized in published works.

The substantial work that has been invested in identifying core narrative elements provides a strong foundation for generating confirmatory research hypotheses. At the same time, many gaps in our knowledge require that exploratory research be conducted as well. And, of course, the two can work together in productive ways. For example, McAdams (2006a) used a 
series of studies on the life stories of highly generative American adults to derive a core set of themes that define what he termed "the redemptive self." Building on this descriptive work, McAdams and Guo (2015) empirically tested the association between these core themes and psychosocial outcomes, demonstrating how individuals use different configurations of narrative identity as resources for meaning. Thus, a key element in narrative study design is developing exploratory questions or hypotheses appropriate to narrative data.

It is also possible to conduct research on narrative identity on data that have not specifically been collected with narrative questions in mind. Many studies include free-response qualitative data, which may lend itself to narrative investigation. For example, Silver and colleagues (2002) collected a dataset designed to examine psychological adaptations following the September 11, 2001 terrorist attacks in the United States. Participants were provided three prompts asking them to write about their experiences. Adler and Poulin (2009) used these responses to look at the emotional sequencing of narratives and their association with psychological well-being. The key question to ask about such existing data is whether it is sufficiently narrative. In other words, were participants telling stories, or were they simply reporting thoughts, feelings, ideas, etc.? There is no commonly-used definition of "narrative," but if the majority of participants are not telling stories - that is, experiences of specific life events that unfold over time - the data do not lend themselves to productive narrative inquiry.

Along those lines, there is a related and essential distinction between narrative and linguistic methods (e.g., Pennebaker, Mehl, \& Neiderhoffer, 2003). Although narratives are comprised of words, their meaning is an emergent property that cannot be reduced to word choice, even though word choice does influence a narrative's meaning. Indeed, studies that have empirically compared narrative and linguistic approaches within the same dataset have failed to 
identify a linguistic substrate of common narrative themes (Weston, et al., 2015), and word count is rarely meaningfully correlated with many narrative themes (Adler et al., 2016). Linguistic methods provide data that may complement those derived from narrative approaches (such as emotional tone, which is often readily apparent in word choices), but are not interchangeable (Weston et al., 2015).

\section{Designing Narrative Prompts}

Obtaining narrative data is dependent on the crafting of narrative prompts, which ought to always be driven primarily by the research questions. One commonly used instrument for collecting extensive narrative data in the personality literature is the Life Story Interview (LSI; McAdams, 2008). This semi-structured interview protocol has been used in dozens of studies and has proven to be an effective vehicle for collecting personal stories. It facilitates selfnarration by tapping the major components of autobiographical memory, lifetime periods (chapters) and more specific episodes (scenes), while also projecting the interviewee's life into the future and providing conceptualizing information on personal values and ideology (e.g., Conway \& Pleydell-Pearce, 2000).

The LSI is a battery consisting of multiple narrative prompts that range from specific domains of individuals' lives such as particular challenges, to “Key Scenes," the important life memories that serve as the core of the protocol. Three prompts that have received extensive attention in research on narrative identity are stories about a life high point, a low point, and a turning point (e.g., Cox \& McAdams, 2014; McLean \& Pratt, 2006). The complete LSI can take 1-2 hours to collect and produces a massive amount of text. Studies seeking to include narrative data, but that do not strive to capture the complete life story, would be well-served by including these three scenes, if they are appropriately geared towards the research questions. 
Some researchers may be interested in more specific life experiences, rather than general categories (e.g., turning points). In such cases, the LSI provides a good template for writing prompts. For example, in studies of topics as diverse as personal transgressions (Mansfield, Pasupathi, \& McLean, 2015), identity content (e.g., politics, gender, family; McLean, Syed, \& Shucard, 2016) the transition to parenthood (Dunlop et al, in press), and career changes and religious conversions (Bauer \& McAdams, 2004), the narrative prompts mirrored those in the LSI. A template for such a prompt is:

Please describe a scene, episode, or specific moment in your life that stands out as [emblematic of the topic of interest]. Please describe this scene in detail. What happened, when and where, who was involved, and what were you thinking and feeling? Also, please say a word or two about why you think this particular moment stands out to you now and what the scene may say about who you are as a person.

Embedded in this prompt are several central elements: a specific, bounded scene, a request for detail and elaboration, and a request for reflection or meaning-making about the scene. Examining specific life experiences, as opposed to the life story, allows researchers to ask questions about the meaning of particular experience and about the ways in which meaningmaking processes differ across experiences (e.g., Mansfield, Pasupathi, \& McLean, 2015).

Prompts may also be designed in ways that differ from the LSI. Whether drawn from the LSI or not, the design of prompts ought to be grounded in the theoretical questions under examination. For example, Singer’s (Blagov \& Singer, 2004) self-defining memory prompt provides an alternative prototype that has been widely used in research on autobiographical memory and narrative identity (e.g., Singer, et al., 2013). Likewise, the Adult Attachment Interview (AAI; Main, George, \& Kaplan, 1985) has been used to elicit narratives that tap the narrative organization of personal experiences with attachment figures (e.g., Waters, Brockmeyer, \& Crowell, 2013). Critically, working with such narratives requires an 
understanding of how the prompt derives from the research question. For example, the LSI turning point prompt assumes the importance of change to identity, whereas a self-defining memory allows more room for the narration of change and stability. In contrast, the AAI assumes non-conscious processes, so the coding systems center on capturing implicit emotional coherence of narration.

Pilot testing newly-written prompts, or standard prompts used with novel samples can ensure that participants have understood the researchers' intentions so that the prompts will elicit the kind of data sought (e.g., Syed, 2015). There is no quantitative metric for evaluating the pilot testing of prompts, but examining pilot data can indicate that participants can answer prompts appropriately and provide code-able narrative data.

Narrative data raise unique ethical issues. First, reporting personal narratives can be a taxing, emotional experience, particularly for certain types of events (e.g., low points, traumas, transgressions). The consent, data collection, and debriefing procedures should be organized with this in mind. It may be especially important to clearly define the task before consent, check in with participants during data collection, and/or be prepared to make referrals during debriefing. Second, narratives cannot be anonymized in the same way other types of data can. Such issues should inform the consent process (by explicating for participants the ways in which their data might be used), the training of raters (by establishing a standard of professionalism and respect in engaging with the data), the handling of the data (by setting clear guidelines for restricting access), and the presentation of results (by striving to mask particular individuals' identity while allowing authentic voices to illustrate broader trends). More broadly, these ethical issues pose challenges to data sharing.

\section{Collecting Narratives}


Narratives may be collected from participants in either oral or written formats. Interview approaches allow for follow-up questioning and tends to produce more elaborated narratives, and can also help to establish rapport and comfort, which may facilitate disclosure. Written approaches may produce briefer and possibly more coherent responses, reduce interviewer effects, and may facilitate the sharing of sensitive information or stories for which participants might desire more anonymity (cf. Grysman \& Denny, in press; McCoy \& Dunlop, 2016).

When collecting oral narratives, interviewers need to be carefully selected for professionalism, as they will need to walk a delicate balance between establishing and maintaining rapport without directing the content of participants’ responses. Training of interviewers, including pilot interviews with feedback, is critical. We encourage interviewers to begin by explaining that they will be using a protocol and need to adhere to it, but will strive to make the interview feel as conversational as possible (Josselson, 2013). In order to assure that participants are exposed to the same type of stimulus, interviewers should refrain from commenting on responses or directing the conversation beyond adhering to the prompts. Remaining engaged listeners and using non-verbal cues to convey their interest are encouraged, and research suggests this will elicit more elaborated responses (Bavelas, Coates, \& Johnson, 2000). Interviewers should also strive to elicit responses to each part of the prompt. For example, participants in studies using the LSI often forget to address the last part of the prompts that asks them to reflect on why the scene stands out as important and what it might say about them as a person. The choice to follow up on this omission depends on the motivating research questions - is it more important to have participants respond to all aspects of the prompt, or are omissions of some parts of the prompts in and of themselves meaningful data? 
Interviews should be recorded, and transcribed precisely; it is not possible to code narratives adequately from audio or video. Many researchers use professional transcription services. If not employing professional services, double-checking all transcriptions is necessary, at minimum. For example, simple deletions like missing the word "not" can transform the meaning of a sentence (“that was not good” could become "that was good”).

Once transcribed, most researchers code from the transcripts. Although vocal intonation and non-verbal behavior reveal aspects of people’s experiences that are not apparent in the words they use, returning to the recording is primarily done to increase precision in coding statements that are otherwise unclear (e.g., "that was great” could mean what it says, or be said with sarcasm). Methods exist for coding non-verbal material as well (e.g., Harrigan, Rosenthal, \& Scherer, 2005), and the field would benefit from systematically investigating these associated components of narration.

Obtaining written narratives is much less time- and labor-intensive for the investigator, but several key points must be kept in mind. First, it is especially important to pilot written narrative prompts, as there will not be an opportunity to ask targeted follow-up questions to ensure participants are providing appropriate data. Second, the setting of a written narrative assessment is also important. We recommend a quiet room, in which the participant can be alone. The presence of others can alter the experience by reducing privacy, creating social comparisons about completion time or process, and merely reminding people of social and selfpresentational concerns. Our experience suggests that written narratives obtained in the lab may be substantially higher in quality than those obtained online. ${ }^{1}$

\footnotetext{
${ }^{1}$ Operationalizing narrative quality is a complex issue and multiple frameworks have been proposed. Most suggest that a narrative ought to have a coherent episodic structure (e.g., McCabe \& Peterson, 1984), include rich detail (e.g., Baron \& Bluck, 2009) including emotional detail (e.g., Baron \& Bluck, 2011), are engaging to listeners/readers (e.g., Baron \& Bluck, 2011), and provide convincing causal explanations for the self (e.g.,
} 
Narratives are typically obtained alongside responses to questionnaire data. There are no established best practices about the order in which data should be collected. It may make sense to collect questionnaire data first if narrative data without questionnaire data would not be useful for analysis. Alternatively, when participation fatigue is a concern, it may make sense to collect narrative data first. Order effects of narratives are also a potential concern, though two published studies have not found effects on other survey measures (e.g., McLean \& Pals, 2008; McLean et al., 2016).

\section{Coding Narratives}

Before narratives are presented to raters they ought to be de-identified and randomized by participant (longer transcripts are typically rated as a set, not randomized by prompt). Deidentification itself can be nuanced (narrative content often includes identifying details), but ensuring that raters are unable to detect any meaningful information about the data that might influence their coding is important. For example, if the focus is on gender differences, researchers may want to remove demographic information for coding, if possible.

Selecting narrative variables for investigation ought to be driven by the hypotheses of the specific study. As noted above, many coding systems have been developed and are readily available for operationalizing these themes within new datasets, such as those listed in Table 1. In some instances, it may be necessary to develop a new coding system for tapping constructs that have not yet been examined. This intensive process is described by Syed and Nelson (2015; see also McAdams et al., 2008). In addition, applying an existing coding system to a new dataset is not always straightforward. For example, if participants are of a substantially different

McAdams, 2006b). McAdams (2006b) adds that the kinds of stories that constitute narrative identity must also advance living action, influencing how we live. Our experience as researchers using narrative methods aligns with research which demonstrates that most individuals within a given culture rely on similar implicit theories about story quality (e.g., Baron \& Bluck, 2011). 
developmental stage or background, or if the prompts are quite different, revision of the existing coding system may be necessary. Although we encourage researchers to continue exploring new narrative variables and developing coding systems for operationalizing them, we simultaneously encourage researchers to explicitly relate new systems to existing ones, in both theoretical and empirical ways (to demonstrate convergent, divergent, and incremental validity) when studying topics that have received prior empirical attention.

While there is variation in the field, the most common unit of analysis for coding is the scene. For example, the LSI contains multiple Key Scenes and typically a single score for each narrative variable being assessed is assigned to each scene. In studies not using the LSI, it is still possible to divide data into scenes, often assigning one score per prompt. This approach allows researchers to conduct between-person analyses at the level of the specific scene (i.e., looking at low points across individuals; e.g., Pals, 2006), or at the level of the individual (i.e., averaging scores across all scenes within an individual to create summary scores for comparison across individuals; e.g., McAdams et al., 2008), or for within-person analyses (i.e., comparing high points to low points; e.g., McLean \& Pals, 2008).

The first step in coding is training raters to a high standard of inter-rater reliability. Before any substantial portion of the data is coded, raters must demonstrate that they are using the coding system(s) in reliable and consistent ways. This training phase is crucial to the scientific soundness of the coding, which will ultimately produce the quantitative representation of the narrative data. As Syed and Nelson (2015) point out, inter-rater reliability is best conceived of as a process and they outline the many decision points in this process. While a set of statistics will represent this procedure in any report of the study, inter-rater reliability informs every step of the coding process. 
Training is typically undertaken by two raters for each narrative variable being assessed. Narrative coding cannot be completed entirely by a single coder, for that would not allow one to determine the degree of inter-rater reliability, and larger group of coders may pose challenges to obtaining inter-rater reliability. Training typically begins by reading the coding system, and discussing it to understand the rationale and what the system aims to capture. Then, coders read through a sub-set of narratives together, discussing how the coding system might be applied. Raters continue this discussion until they reach a growing consensus about the process. During this phase, the coding system may be refined, making additional notations about emerging idiosyncrasies from the match between this particular coding system and this particular dataset.

Then, raters individually score a new sub-set of narratives. These scores are then compared statistically to determine whether inter-rater reliability has been achieved. There are different approaches to calculating inter-rater reliability, depending on the nature of the coding systems (see Syed \& Nelson 2015). Raters rarely achieve appropriate inter-rater reliability on the first attempt, in which case they should discuss disagreements, come to consensus for those data points, refine the coding system if necessary, and then attempt another set of coding independently. This often takes $10-25 \%$ of the dataset, depending on its size, and the frequency of codes (e.g., codes with low base rates may need more narratives to achieve appropriate reliability).

Once an adequate degree of inter-rater reliability is achieved, raters either split the remaining data and code independently, or two raters proceed with coding the remainder of the data (either generating consensus codes or taking means of any discrepancies). During this phase of largely independent coding, it is important to have regular discussions with the raters in order to prevent deviations from the coding system (referred to as “drift;” Wolfe, Moulder, \& 
Myford, 2000). Early on in the independent coding, raters are encouraged to bring any examples that they feel uncertain about to the team for discussion and consensus rating (or averaging). Typically, the number of such examples decreases dramatically throughout the coding process. It is optimal to repeat the formal reliability phase of coding to ensure appropriate inter-rater reliability is maintained throughout the coding process, but at minimum periodic checks should be made to avoid coder drift.

If multiple narrative variables are going to be assessed by the same raters, it is appropriate to code the entire dataset for a single variable before coding another variable. This allows raters to remain in a focused mindset when reading the transcripts, as coding can be like viewing a narrative through a specific lens, seeking to filter the complex material to reveal only certain aspects. Multiple coding teams may be used for examining different themes in the same set of narratives as long as inter-rater reliability is established and maintained within rater teams.

While coding is underway, we recommend that raters keep track of emblematic examples from the data to represent each coding system (high/low poles of each system, or quotes that exemplify an instance-based/categorical system). This is helpful for two reasons. First, when preparing the Method section of a manuscript, it is vital to include quotes that illustrate how the coding system was applied to this dataset in both didactic (explaining the mechanics of the system) and illustrative (sharing actual quotes) ways. Second, from a theoretical perspective, it is essential not to let the presentation of the study stray too far from the actual voices of the participants, so evocative quotes serve to ground the statistics in the stories they seek to describe or explain.

Regardless of how engaging a particular sample of narratives may be, coder burnout may become an issue. Quality coding takes a high degree of attention and close reading, and can also 
be emotionally taxing, depending on the type of narratives. We encourage researchers to intersperse the coding process with other research tasks to keep raters engaged and focused when analyzing narratives, and to set limits on the amount of time for any one coding session.

Once all coding is complete, the narrative data may be reconnected with other data from participants and inferential statistics can be applied to address the central questions of the study, typically involving the relationships between narrative variables and other variables of interest.

\section{Conclusion, Limitations, and Future Directions}

This primer has outlined the most common approach to empirical quantitative research with narrative methods. Our hope is that it will both encourage scholars to include narratives in their research and serve as a practical tool to guide their initial forays into the field. In addition to the many theoretical reasons for using narrative methods, narrative data are fundamentally generative. A study that includes only responses to questionnaires, laboratory tasks, or psychophysiological data can only ask so many questions, but narratives offer a nearly endless opportunity for revisiting and for discovery.

However, there are limitations to taking a narrative approach. As should be clear from this review, it is time and labor intensive to collect and code narratives. It is an approach that also demands a certain facility with language, and work for researchers to ensure that the prompts and questions are personally and culturally relevant to participants. Thus, given the time and effort required of narrative coding, we encourage researchers to first ask whether this is the most effective way of tapping a construct of interest. In making that decision, the topic of incremental validity is especially important: will examining narrative data provide explanatory power not adequately captured by other methods (Adler et al., 2016)? In addition, it is essential to remember that quantitative coding is not always the appropriate approach either. Many vital 
questions in the study of narrative identity beg for inductive, qualitative methods that can reveal complexity and explore new avenues better than that could be obtained by relying on existing coding systems (cf., Hammack, 2010; Jossleson, 2009). Such research makes an important contribution to the literature on narrative identity itself, as well as sometimes lays a strong foundation for subsequent quantitative work (e.g., Syed, 2015).

There are many directions in which researchers can take the narrative approach, many of which can go well beyond the focus on narrative identity, which we have elaborated here. For example, we suggest that the prompts regarding specific experiences, such as the transition to parenthood, can be applied to all kinds of psychologically meaningful phenomena. Researchers interested in learning more about the content and meaning of the phenomena on which their programs of research focus can employ narrative methods to elucidate such phenomena. That is, narrative can be a powerful tool for studies that are not designed with narrative identity as the primary focus.

One other direction that we emphasize is the utility of narrative for understanding the cultural context of the individual. Given the inherent descriptive quality of an individual's story, it is a rich source of information about the culture and context of personality and development. Many coding systems and studies have aimed to capture the individual processes of personal development, but there is an opportunity to expand that lens to the cultural context in which identity and personality develops - from the particularities of experience for members of a cultural group, to the norms about what experiences qualify as positive or negative, to the cultural structures and formats for narrating such experiences (e.g., Hammack, 2008; McLean \& Syed, 2016; Syed \& Azmita, 2010). Expanding this approach to more diverse groups and cultures will take a great deal of work in terms of ensuring that the prompts are appropriate, but 
could reveal aspects of unique culturally-situated meaning-making experiences that are not as easily grasped by surveys and which complement the results of ethnographic work.

This primer has sought to make explicit the most common research practices using narrative methods that have been used during this period of dramatic growth in the field. Without a doubt, the field will continue to evolve. A publication such as this is fundamentally a static document, but the nine authors of this paper welcome questions, feedback, and recommendations from scholars who use it as we collectively seek to continually improve the field. 


\section{References}

Adler, J.M. (2012). Living into the story: Agency and coherence in a longitudinal study of narrative identity development and mental health over the course of psychotherapy. Journal of Personality and Social Psychology, 102(2), 367-389.

Adler, J.M., Lodi-Smith, J., Philippe, F.L., Houle, I. (2016). The incremental validity of narrative identity in predicting well-being: A review of the field and recommendations for the future. Personality and Social Psychology Review, 20(2), 142-175.

Adler, J.M. \& Poulin, M. (2009). The political is personal: Narrating 9/11 and psychological well-being. Journal of Personality, 74(4), 903-932.

Adler, J.M., Turner, A.F., Brookshier, K.M., Monahan, C., Walder-Biesanz, I., Harmeling, L.H., Albaugh, M., McAdams, D.P., Oltmans, T.F. (2015). Variation in narrative identity is associated with trajectories of mental health over several years. Journal of Personality and Social Psychology, 108(3), 476-496.

Baron, J. M., \& Bluck, S. (2009). Autobiographical memory sharing in everyday life: Characteristics of a good story. International Journal of Behavioral Development, 33, 105117.

Baron, J. M., \& Bluck, S. (2011). That was a good story! Preliminary construction of the Perceived Story Quality Index. Discourse Processes, 48(2), 93-118.

Bauer, J. J., \& McAdams, D. P. (2004). Personal growth in adults’ stories of life transitions. Journal of Personality, 72, 573-602.

Bavelas, J.B., Coates, L., \& Johnson, T. (2000). Listeners as co-narrators. Journal of Personality and Social Psychology, 79(6), 941-952. 
Blagov, P. \& Singer, J.A. (2004). Four dimensions of self-defining memories (specificity, meaning, content, and affect) and their relationship to self-restraint, distress, and repressive defensiveness. Journal of Personality, 72(3), 481-511.

Conway, M. A. \& Pleydell-Pearce, C. W. (2000). The construction of autobiographical memories in the self-memory system. Psychological Review, 107, 261-288.

Cox, K. S., \& McAdams, D. P. (2014). Meaning making during high and low point story episodes predicts emotion regulation two years later: How the past informs the future. Journal of Research in Personality, 50, 66-70.

Dunlop, W. L., Walker, L. J., Hanley, G. E., \& Harake, N. (in press). The psychosocial construction of parenting: An examination of parenting goals and narratives in relation to well-being. Journal of Happiness Studies.

Dunlop, W.L. \& Tracy, J.L. (2013). Sobering stories: Narratives of self-redemption predict behavioral change and improved health among recovered alcoholics. Journal of Personality and Social Psychology, 104, 576 - 590.

Fivush, R. (2010). Speaking Silence: The social construction of voice and silence in cultural and autobiographical narratives. Memory, 18, 88-98.

Fivush, R. \& Merrill, N. (2016). An ecological systems approach to family narratives. Memory Studies, 9, 305-14.

Grysman, A. \& Denney, A. (in press). Gender, experimenter gender and medium of report influence the content of autobiographical memory report. Memory.

Grysman, A., Merrill, N., \& Fivush, R. (2016). Gender differences in autobiographical memory: A mediated moderation approach to an elusive finding. Memory. 
Habermas, T., \& Reese, E. (2015). Getting a life takes time: The development of the life story in adolescence, its precursors and consequences. Human Development, 58, 172-201.

Hammack, P.L. (2008). Narrative and the cultural psychology of identity. Personality and Social Psychology Review, 12(3), 222-247.

Hammack, P.L. (2010). The political psychology of personal narrative: The case of Barack Obama. Analyses of Social Issues and Public Policy, 10(1), 182-206.

Harrigan, J. A., Rosenthal, R., \& Scherer, K.R. (2005). New handbook of methods in nonverbal behavior research. New York: Oxford University Press.

Jossleson, R. (2009). The present of the past: Dialogues with memory over time. Journal of Personality, 77(3), 647-668.

Josselson, R. (2013). Interviewing for qualitative inquiry: A relational approach. Guilford Press. Josselson, R. \& Lieblich, A. (1993). The Narrative Study of Lives. Thousand Oaks, CA: Sage. Lodi-Smith, J., Geise, A.C., Roberts, B.W., \& Robins, R.W. (2009). Narrating personality change. Journal of Personality and Social Psychology, 96, 679-689.

Main, M., George, C., \& Kaplan, N. (1985). Adult attachment interview. Growing points of attachment theory. Monographs of the Society for Research in Child Development.

Mansfield, C.D., Pasupathi, M., \& McLean, K.C. (2015). Is narrating growth in stories of personal transgressions associated with increased well-being, self-compassion, and forgiveness of others? Journal of Research in Personality, 58, 69-83.

Maruna, S. (2001). Making good: How ex-convicts reform and rebuild their lives. Washington, DC: American Psychological Association.

McAdams, D. P. (1995). What do we know when we know a person? Journal of Personality, 63, 363-396. 
McAdams, D.P. (2006a). The redemptive self: Stories Americans live by. New York: Oxford University Press.

McAdams, D. P. (2006b). The problem of narrative coherence. Journal of Constructivist Psychology,19, 109-112.

McAdams, D.P. (2008). The LSI. The Foley Center for the Study of Lives, Northwestern University, Evanston, IL. Retrieved at: http://www.sesp.northwestern.edu/foley/instruments/interview/

McAdams, D. P., Albaugh, M., Farber, E., Daniels, J., Logan, R. L., \& Olson, B. (2008). Family Metaphors and Moral Intuitions: How Conservatives and Liberals Narrate Their Lives. Journal of Personality and Social Psychology, 978-990.

McAdams, D. P., \& Guo, J. (2015). Narrating the generative life. Psychological Science, 26, 475-483.

McAdams, D. P., Hanek, K. J., \& Dadabo, J. G. (2013). Themes of self-regulation and selfexploration in the life stories of religious American conservatives and liberals. Political Psychology, 34(2), 201-219.

McAdams, D.P. \& McLean, K.C. (2013). Narrative identity. Current Directions in Psychological Science, 22(3), 233-238.

McAdams, D. P., \& Pals, J. L. (2006). A new Big Five: Fundamental principles for an integrative science of personality. American Psychologist, 61, 204-217.

McCabe, A., \& Peterson, C. (1984). What makes a good story? Journal of Psycholinguistic Research,13, 457-480.

McCoy, T. P. \& Dunlop, W. L. (2016). Contextualizing narrative identity: A consideration of assessment settings. Journal of Research in Personality, 65, 16-21. 
McLean, K.C. (2015). The co-authored self: Family stories and the construction of personal identity. New York: Oxford University Press.

McLean, K. C., \& Pals, J. L. (2008). Reminiscence functions: Emotional valence, age and wellbeing. Memory, 751-672.

McLean, K. C., Pasupathi, M., Fivush, R., Grenhoot, A. F., \& Wainryb, C. (2016). Does within person variability in narration matter and for what? Journal of Research in Personality.

McLean, K. C., Pasupathi, M., \& Pals. J. L. (2007). Selves creating stories creating selves: A process model of narrative self development in adolescence and adulthood. Personality and Social Psychology Review, 11, 262-278.

McLean, K. C., \& Pratt, M. W. (2006). Life’s little (and big) lessons: Identity statuses and meaning-making in the turning point narratives of emerging adults. Developmental Psychology, 42, 714-722.

McLean, K. C. \& Syed, M. (2016). Personal, master, and alternative narratives: An integrative framework for understanding identity development in context. Human Development, 58, 218349.

McLean, K. C., Syed, M., \& Shucard, H. (2016). Gender Identity Development in Emerging Adulthood: A Master Narrative Approach. Emerging Adulthood.

McLean, K. C., \& Thorne, A. (2003). Late adolescents’ self-defining memories about relationships. Developmental Psychology, 39, 635-645.

Pals, J. L. (2006). Narrative identity processing of difficult life experiences: Pathways of personality development and positive self-transformation in adulthood. Journal of Personality, 74, 1079-1110. 
Pasupathi, M., Billitteri, J., Mansfield, C.D., Wainryb, C., Hanley, G.E., \& Taheri, K. (2015).

Regulating emotion and identity by narrating harm. Journal of Research in Personality, 58, 127-136.

Pennebkaer, J.W., Mehl, M.R., \& Niederhoffer, K.G. (2003). Psychological aspects of natural language use: Our words, our selves. Annual Review of Psychology, 54, 547-577.

Silver, R.C., Holman, E.A., McIntosh, D.N., Poulin, M., \& Gil-Rivas, V. (2002). Nationwide longitudinal study of psychological responses to September 11. JAMA, 288(10), 12351244.

Singer, J. A. (2004). Narrative identity and meaning-making across the lifespan: An introduction. Journal of Personality, 72, 437-460.

Singer, J. A., Blagov, P., Berry, M., \& Oost, K. M. (2013). Self-defining memories, scripts, and the life story: Narrative identity in personality and psychotherapy. Journal of Personality, 81(6), 569-582.

Syed, M. (2015). Theoretical and methodological contributions of narrative psychology to ethnic identity research. In C. Santos \& A. J. Umaña-Taylor (Eds.). Studying ethnic identity: Methodological advances and considerations for future research (pp. 27-54). Washington, DC: American Psychological Association.

Syed, M., \& Azmitia, M. (2010). Narrative and ethnic identity exploration: A longitudinal account of emerging adults’ ethnicity-related experiences. Developmental Psychology, 46(1), 208-219.

Syed. M. \& Nelson, S.C. (2015). Guidelines for establishing reliability when coding narrative data. Emerging Adulthood, 3(6), 375-387. 
Waters, T. E. A, Brockmeyer, S., \& Crowell, J.A. (2013). AAI coherence predicts caregiving and care seeking behavior in couple problem solving interactions: Secure base script knowledge helps explain why. Attachment and Human Development, 15(3), 316-331.

Weston, S.J., Cox, K.S., Condon, D.M., \& Jackson, J.J. (in press). A comparison of human narrative coding of redemption and automated linguistic analysis for understanding life stories. Journal of Personality.

Wolfe, E. W., Moulder, B. C., \& Myford, C. M. (2000). Detecting differential rater functioning over time (DRIFT) using a Rasch multi-faceted rating scale model. Journal of Applied Measurement, 2, 256-280. 
Table 1. Commonly-assessed narrative variables (a representative set)

\begin{tabular}{|c|c|c|}
\hline $\begin{array}{l}\text { Narrative Category } \\
\text { (Adler, Lodi- } \\
\text { Smith, Philippe, \& } \\
\text { Houle, 2016) }\end{array}$ & $\begin{array}{l}\text { Narrative } \\
\text { Variable }\end{array}$ & Definition \\
\hline \multirow[t]{3}{*}{$\begin{array}{l}\text { Motivational } \\
\text { Themes }\end{array}$} & Agency* & $\begin{array}{l}\text { The degree to which the protagonist can initiate } \\
\text { changes on his or her own, achieve some degree of } \\
\text { control over the course of his or her experiences, and } \\
\text { affect his or her own life. This is often exemplified } \\
\text { by aspects of self-mastery and control, status } \\
\text { striving, achievement, and power. }\end{array}$ \\
\hline & Communion* & $\begin{array}{l}\text { The degree to which the protagonist aims to have a } \\
\text { sense of togetherness and harmony with other people } \\
\text { or their environment, to share, help, connect to, and } \\
\text { care for others. }\end{array}$ \\
\hline & Growth Goals & $\begin{array}{l}\text { The degree to which the protagonist makes } \\
\text { intentional efforts to guide his or her self- } \\
\text { development in a personally meaningful direction } \\
\text { oriented toward personal growth. }\end{array}$ \\
\hline \multirow[t]{3}{*}{ Affective Themes } & Contamination* & $\begin{array}{l}\text { The protagonist describes an event that moves from } \\
\text { a good, affectively positive scene to a bad, } \\
\text { affectively negative scene, in which good things turn } \\
\text { into bad outcomes. }\end{array}$ \\
\hline & Redemption* & $\begin{array}{l}\text { The protagonist describes an event that moves from } \\
\text { a bad, affectively negative scene to a subsequent } \\
\text { good, affectively positive scene. The bad is } \\
\text { redeemed, salvaged, mitigated, or made better in } \\
\text { light of the ensuing good. }\end{array}$ \\
\hline & $\begin{array}{l}\text { Positive } \\
\text { Resolution* }\end{array}$ & $\begin{array}{l}\text { The protagonist is able to come to peace with, or let } \\
\text { go of, a challenging event. }\end{array}$ \\
\hline \multirow[t]{3}{*}{$\begin{array}{l}\text { Themes of } \\
\text { Integrative } \\
\text { Meaning }\end{array}$} & $\begin{array}{l}\text { Accommodative } \\
\text { Processing }\end{array}$ & $\begin{array}{l}\text { The degree to which the protagonist has been forced } \\
\text { to change, centrally and qualitatively, his or her } \\
\text { views of the self and world, by actively experiencing } \\
\text { a paradigmatic shift that requires a revision of } \\
\text { structures and/or important changes in response to } \\
\text { the environment. }\end{array}$ \\
\hline & $\begin{array}{l}\text { Exploratory } \\
\text { Processing* }\end{array}$ & $\begin{array}{l}\text { The degree to which the protagonist makes an active } \\
\text { and engaged effort to explore, reflect on, or analyze } \\
\text { a difficult experience with openness to learning from } \\
\text { it and incorporating a sense of change into the life } \\
\text { story. }\end{array}$ \\
\hline & $\begin{array}{l}\text { Meaning- } \\
\text { Making* }\end{array}$ & $\begin{array}{l}\text { The degree to which the protagonist learns } \\
\text { something or gleans a message from an event (e.g., } \\
\text { no meaning, vague meaning, learning concrete } \\
\text { lessons, gaining deep insights). }\end{array}$ \\
\hline
\end{tabular}




\begin{tabular}{ll}
$\begin{array}{l}\text { Structural } \\
\text { Elements of } \\
\text { Narratives }\end{array}$ & $\begin{array}{l}\text { The degree to which the narrator situates the } \\
\text { characters of his story and their actions in a specific } \\
\text { context, the story follows a temporal sequence of } \\
\text { goal-oriented actions that are culturally recognized, } \\
\text { emotions are clearly expressed in support of the } \\
\text { point of the narrative, and narrative is integrated into } \\
\text { larger life themes and meanings. } \\
\text { The degree of engagement in the narrative } \\
\text { processing, as shown by depth of thought and } \\
\text { nuance, such as seeing a variety of perspectives or } \\
\text { emotions. }\end{array}$ \\
\hline
\end{tabular}

Note: This table was adapted from Table 2 in Adler, Lodi-Smith, Philippe, \& Houle (2016) and from Table 1in McAdams \& McLean (2013). Asterisks indicate that the variable appeared in both sources. 
Phases of Narrative Research

Asking Narrative

Questions

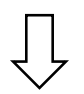

Write or Identify

Narrative Prompt(s)

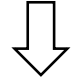

Determine Data

Collection Context

$\sqrt{5}$

Prepare Narratives for Coding

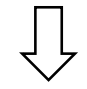

Develop Coding Manual<smiles>C1CCCCC1</smiles>

Coding Process<smiles>C1CCC1</smiles>

Data Reporting

\section{Major Considerations}

- Develop testable hypotheses that pose narrative questions

- Develop exploratory research questions

- Interview participants

- Written or typed narratives

- Use existing prompt(s)

- Develop new prompt(s)

- Life Story Interview as template

- Transcribe audio/video or handwritten narratives

- De-identify the data as necessary

- Determine the unit of analysis (e.g., scene, person, utterance)

- Adapt existing coding manual; assess suitability

- Develop new coding manual

- Train raters who are blind to study hypotheses

- Revise manual as necessary

- Establish initial inter-rater reliability using appropriate statistic prior to coding complete dataset

- Code all data, assessing rater drift

- Descriptive and inferential statistics, as appropriate

- Inclusion of illustrative cases and negative cases

Figure 1. Overview of the narrative research process 